\title{
Is iPLA $\beta$ a Novel Target for the Development of New Strategies to Alleviate Inflammatory Bowel Disease?
}

\author{
Toni Petan ${ }^{1}$ - Igor Križaj ${ }^{1,2}$
}

Published online: 21 September 2015

(c) Springer Science+Business Media New York 2015

Phospholipases $\mathrm{A}_{2}\left(\mathrm{PLA}_{2} \mathrm{~s}\right)$, lipolytic enzymes that release fatty acids (FAs) and lysophospholipids from the hydrolysis of the $s n-2$ ester bond of membrane glycerophospholipids, modulate (phospho)lipid-related homeostatic and stimulus-induced intracellular processes, linking lipid metabolism with cell signaling [1,2]. Although the diverse products released by their activity on cell membranes have signaling and metabolic activities in and of themselves, the products may be further converted into hundreds of lipid mediator molecules, including arachidonic acid (AA)derived eicosanoids and $\omega-3$ polyunsaturated FA-derived pro-resolving mediators [3]. Unsurprisingly, $\mathrm{PLA}_{2} \mathrm{~S}$ are involved in diverse and fundamental biological processes, including inflammation, immunity, reproduction, atherosclerosis, cancer, and neurodegeneration [2]. Among the human $\mathrm{PLA}_{2} \mathrm{~S}$, eleven members belong to the secreted $\mathrm{PLA}_{2}\left(\mathrm{sPLA}_{2}\right)$ family of low-molecular-mass $\mathrm{Ca}^{2+}$-dependent enzymes that function in the extracellular space on cell membranes, lipoproteins, bacteria, microparticles, and viruses [3, 4]. Most $\mathrm{PLA}_{2} \mathrm{~S}$ are, however, intracellular enzymes, including the $\mathrm{Ca}^{2+}$-dependent cytosolic $\mathrm{PLA}_{2} \mathrm{~S}$ $\left(\mathrm{CPLA}_{2} \mathrm{~S}\right.$ or group IV $\mathrm{PLA}_{2} \mathrm{~s}$ ) and the $\mathrm{Ca}^{2+}$-independent $\mathrm{PLA}_{2} \mathrm{~s}$ ( $\mathrm{iPLA}_{2} \mathrm{~S}$ or group VI PLA $2 \mathrm{~s}$ ). Among the nine enzymes within the $\mathrm{PLA}_{2}$ family, also named the patatin-

Toni Petan

toni.petan@ijs.si

$\triangle$ Igor Križaj

igor.krizaj@ijs.si

1 Department of Molecular and Biomedical Sciences, Jožef Stefan Institute, Jamova 39, 1000 Ljubljana, Slovenia

2 Department of Chemistry and Biochemistry, Faculty of Chemistry and Chemical Technology, University of Ljubljana, Večna pot 113, 1000 Ljubljana, Slovenia like phospholipase domain-containing lipase (PNPLA) family, iPLA $_{2} \beta$ (also PNPLA9 or group VIA $\mathrm{PLA}_{2}$ ) is far and away the most studied enzyme [5].

The human $\mathrm{iPLA}_{2} \beta$ gene (PLA2G6) may undergo several alternative splicing events that generate isoforms with varying enzymatic activity and function [6]. The "classic" isoform of $\mathrm{iPLA}_{2} \beta$ (group VIA-1 $\mathrm{PLA}_{2}$ ) is an $85-\mathrm{kDa}$ enzyme with a Ser-Asp catalytic dyad, a conserved glycine-rich, nucleotide-binding motif (GXGXXG) and eight $\mathrm{N}$-terminal ankyrin repeats [5]. The cellular activity of ${ }_{i P L A} \beta$ is regulated by ATP binding, oligomerization, binding to calmodulin, caspase-3-mediated proteolytic cleavage, and alternative splicing $[5,6]$. In contrast to $\mathrm{cPLA}_{2}, \mathrm{iPLA}_{2} \beta$ is not specific for AA, hydrolyzing phospholipids with no preference for their FA substituents or head groups. The biological activity of $\mathrm{iPLA}_{2} \beta$ has been traditionally described as homeostatic membrane remodeling, which includes deacylation/reacylation cycles important for AA incorporation into phospholipids, for phosphatidylcholine (PC) turnover and cell growth, but also for excision-repair mechanisms that maintain mitochondrial membrane integrity [5]. However, recent studies have revealed a number of cell signaling-based roles for the enzyme, such as the regulation of endoplasmic reticulum (ER) stress, apoptosis, cell proliferation, differentiation, and immune cell function [5]. Analyses of mutant mouse models and clinical phenotypes have provided important insights into its (patho)physiological effects, which include osteoblastic differentiation, male fertility, $\beta$-cell function, cancer, and neurodegeneration [5].

In this issue of Digestive Diseases and Sciences, Jiao et al. [7] report a novel function for $\operatorname{iPLA}_{2} \beta$ in the maintenance of intestinal homeostasis. The authors reported that ${ }_{i P L A} \beta$ deficiency significantly exacerbates pathological features in an experimental mouse model of dextran 
sodium sulfate (DSS) colitis. Comparative analyses of wild-type and $\mathrm{iPLA}_{2} \beta$-null mice revealed that $\mathrm{iPLA}_{2} \beta$ deficiency (1) elevates the frequency of intestinal epithelial cell (IEC) apoptosis, (2) suppresses crypt cell proliferation and epithelial recovery from injury, (3) diminishes the number of goblet cells with resultant thinning of the mucus barrier, and (4) increases the inflammatory response to DSS (Fig. 1). Importantly, in support of the enzyme's putative physiological functions, the authors reported that $\mathrm{iPLA}_{2} \beta$ is expressed in human IECs and in immune cells in the normal colon. Since the maintenance of intestinal homeostasis depends on a complex regulatory system that coordinates the interactions between the microbiota, the intestinal epithelium, and the host immune system, a breakdown of this network may lead to the development of inflammatory bowel disease (IBD) [8]. The highly regulated process of IEC turnover includes the removal of old and damaged cells through apoptosis, followed by cell shedding or engulfment by immune cells, which is balanced by ongoing proliferation of stem and progenitor cells, giving rise to new IECs that differentiate and migrate to replace the old cells [9]. The results of Jiao et al. [7] suggest that $\mathrm{PLLA}_{2} \beta$ is

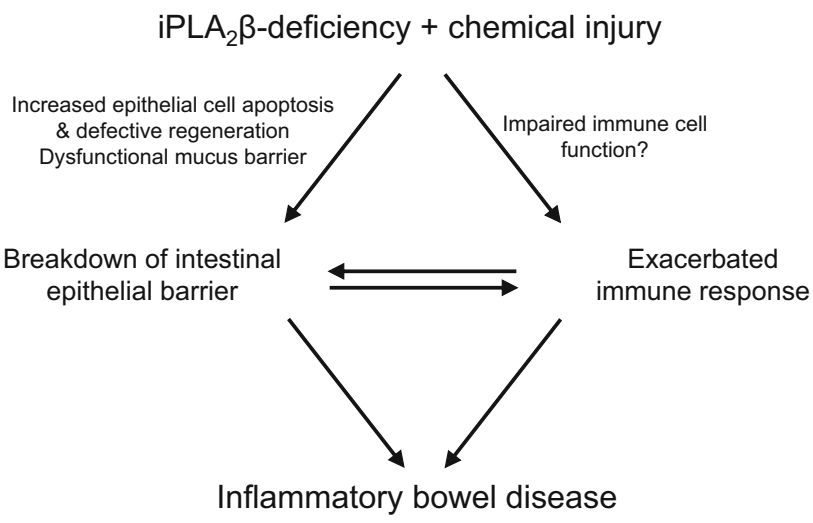

Fig. 1 Proposed contribution of ${ }_{i P L A} \beta$ to the maintenance of intestinal homeostasis. Employing a mouse model of chemically induced colitis, Jiao et al. [7] suggest a new biological function for iPLA $_{2} \beta$-maintenance of intestinal homeostasis. They demonstrate that this enzyme is crucially important for the preservation of epithelial barrier integrity and innate immune response in the mouse colon and ileum. Genetic ablation of $\mathrm{iPLA}_{2} \beta$ in mice reduces their ability to cope with chemically induced colitis, with resultant dysfunction of the intestinal epithelial barrier due to increased IEC apoptosis, suppressed proliferation of crypt cells, impaired epithelial recovery, and a reduced number of goblet cells with a thinner protective mucus layer. This process may be sequentially or concurrently exacerbated by direct (absence of endogenous iPLA ${ }_{2} \beta$ activity in immune cells) or indirect (iPLA ${ }_{2} \beta$ deficiency in epithelial cells) suppressive effects on immune cell function, e.g., reducing the ability of macrophages to efficiently remove apoptotic cell remains. As a consequence of epithelial barrier dissipation, enabling increased transmucosal infiltration of bacteria and associated with cytokine and chemokine overexpression, intestinal inflammation in $\mathrm{PLA}_{2} \beta$-null mice is substantially increased involved in processes that regulate the renewal of the intestinal epithelial barrier and its integrity. How iPLA $2 \beta$ promotes epithelial renewal and barrier integrity, although unknown, may be a consequence of its action at different steps of the cellular turnover process, in any of the different types of colonic epithelial cells or in neighboring immune cells. For example, as the authors suggest, iPLA $\beta$ deficiency may exacerbate DSS-induced apoptosis in stem or progenitor cells or in mature IECs, but also may indirectly suppress cellular renewal mechanisms by preventing efficient removal of apoptotic cells by the immune cells.

Indeed, depending on the cell type, $\mathrm{iPLA}_{2} \beta$ activity stimulates cellular proliferation and, interestingly, induces or prevents apoptosis [5, 6]. Its pro-proliferative activity, present in cells derived from smooth muscle, endothelium, insulin-producing tumors, and colorectal adenocarcinomas, is associated with the production of mitogenic lipid mediators [5]. Yet, iPLA $\beta$ activation also occurs during cellular stress responses where it participates in the execution of the cell death program. In pancreatic $\beta$-cells, $\mathrm{iPLA}_{2} \beta$ supports ER-stress-induced apoptosis by promoting the production of ceramides [5]. On the contrary, $\mathrm{iPLA}_{2} \beta$ facilitates $\beta$-cell survival after lipotoxic stress by executing an excisionrepair mechanism of oxidized cardiolipin that restores the integrity of the mitochondrial membrane [10]. This dual function of $\mathrm{iPLA}_{2} \beta$ in $\beta$-cells is consistent with the thought that the enzyme's activity is organelle and stimuli specific, protecting or damaging tissues according to context [5]. Interestingly, iPLA $2 \beta$-derived lipid mediators also promote ER-stress-mediated $\beta$-cell apoptosis by triggering alternative splicing events that shift the balance between two variants of the Bcl-2 family member Bcl-x in favor of its pro-apoptotic $\mathrm{Bcl}-\mathrm{x}(\mathrm{S})$ form [11]. This mechanism supports the hypothesis that $\mathrm{iPLA}_{2} \beta$ activity may fine-tune the apoptotic program depending on the cellular context. Since $\mathrm{iPLA}_{2} \beta$ may affect cell fate in many ways, it is likely that its activity in the intestinal epithelium may also be associated with different cell- and context-dependent outcomes. Although the results of Jiao et al. [7] suggest that the induction of IEC apoptosis in DSS-treated $\mathrm{iPLA}_{2} \beta$-null mice is not associated with ER stress or with changes in the ratio of $\mathrm{Bcl}-\mathrm{x}$ alternative transcripts, more studies are warranted to clarify how iPLA $\beta$ affects IEC apoptosis in colitis.

The authors also show that $\mathrm{iPLA}_{2} \beta$ deficiency increases the DSS-induced innate immune response in the colon, characterized by infiltrating macrophages and dendritic cells and elevated amounts of pro-inflammatory cytokines [7]. The caspase- 3 truncated form of $\operatorname{iPLA}_{2} \beta$ in apoptotic cells produces lysophosphatidylcholine (LPC), a potent chemoattractant for monocytic cells that then engulf apoptotic cell remains [5]. It is possible that the lower amount of the LPC "eat me" signal in iPLA $2 \beta$-null mice 
[7] leads to an inefficient removal of apoptotic IECs, with resultant secondary necrosis and increased inflammation. Interestingly, the caspase-3-truncated form of $\mathrm{iPLA}_{2} \beta$ also produces mitogenic AA metabolites and lysophosphatidic acid (LPA) that may concurrently provide survival and proliferative signals to neighboring cells [6]. While the increase in the inflammatory response in DSS-treated iPLA $A_{2} \beta$-null mice may be secondary to IEC apoptosis and to the breakdown of the epithelial barrier with resultant uncontrolled microbial infiltration, it is possible that iPLA $_{2} \beta$ deficiency in the immune cells is deleterious for the DSS-challenged colon. Indeed, macrophages lacking $\mathrm{iPLA}_{2} \beta$ display reduced adhesion, spreading, retention, and clearance from inflammation sites [5]. In future studies, it will be important to determine whether iPLA $2 \beta$ activity in immune cells or in epithelial cells is central for its effects on epithelial barrier integrity.

Since $\mathrm{PLA}_{2}$ enzymes often function sequentially or together in a biological process, involvement of other intestinal $\mathrm{PLA}_{2} \mathrm{~S}$ will need to be addressed in future studies of $\mathrm{iPLA}_{2} \beta$ and IBD. Candidates include the group IIA sPLA $_{2}$ whose abundance and activity is not only elevated in the colonic mucosa and the serum of IBD patients, but is also implicated in attenuating colon tumourigenesis [1, 2]. The group $\mathrm{X}$ sPLA 2 , highly expressed in the gastrointestinal tract, participates in gut homeostasis, AA-metabolism, and colon tumourigenesis [2]. Furthermore, both $\mathrm{sPLA}_{2} \mathrm{~s}$, expressed in Paneth cells, have potent bactericidal activity and therefore likely regulate the nature of the microbiota. Polymorphisms in the PLA2G2E gene are associated with ulcerative colitis [3], while the group IID $\mathrm{SPLA}_{2}$ is implicated in protection in a mouse model of the disease [2]. Finally, the absence of the group IVA cPLA enzyme, the principal $\mathrm{PLA}_{2}$ that regulates AA-derived eicosanoid synthesis, is associated with severe intestinal ulceration in mice and humans, most likely due to a decreased synthesis of protective prostaglandin mediators $[2,12]$.

To confirm the above-described findings, made in a chemically induced model of colitis in which $\mathrm{iPLA}_{2} \beta$ expression was genetically modified, it will be necessary to study how iPLA $\beta$ functions under conditions of spontaneous IBD development in different animal models and relevant clinical settings. Given that the increasing amount of evidence supporting the involvement of $\mathrm{iPLA}_{2} \beta$ in apoptosis, ER stress, and autophagy [5] and that dysregulation of these processes is one of the major contributors to disruption of the IE barrier in IBD patients, this study provides a new impetus for studying how iPLA ${ }_{2} \beta$ and other $\mathrm{PLA}_{2} \mathrm{~S}$ regulate cell fate and homeostasis in the gastrointestinal tract.

\section{References}

1. Dennis EA, Cao J, Hsu Y-H, Magrioti V, Kokotos G. Phospholipase $A_{2}$ enzymes: physical structure, biological function, disease implication, chemical inhibition, and therapeutic intervention. Chem Rev. 2011;111:6130-6185.

2. Murakami M, Taketomi Y, Miki Y, Sato H, Hirabayashi T, Yamamoto K. Recent progress in phospholipase $A_{2}$ research: from cells to animals to humans. Prog Lipid Res. 2011; 50:152-192.

3. Murakami M, Sato H, Miki Y, Yamamoto K, Taketomi Y. A new era of secreted phospholipase $\mathrm{A}_{2} . J$ Lipid Res. 2015; 56:1248-1261.

4. Lambeau G, Gelb MH. Biochemistry and physiology of mammalian secreted phospholipases $\mathrm{A}_{2}$. Аnпu Rev Biochem. 2008; 77:495-520.

5. Ramanadham S, Ali T, Ashley JW, Bone RN, Hancock WD, Lei $\mathrm{X}$. Calcium-independent phospholipases $\mathrm{A}_{2}\left(\mathrm{iPLA}_{2} \mathrm{~s}\right)$ and their roles in biological processes and diseases. J Lipid Res. 2015; jlr.R058701.

6. Lei X, Barbour SE, Ramanadham S. Group VIA $\mathrm{Ca}^{2+}$-independent phospholipase $\mathrm{A}_{2}\left(\mathrm{iPLA}_{2} \beta\right)$ and its role in $\beta$-cell programmed cell death. Biochimie. 2010;92:627-637.

7. Jiao L, Inhoffen J, Gan-Schreier H, Tuma-Kellner S, Stremmel $\mathrm{W}$, Sun $\mathrm{Z}$, et al. Deficiency of group VIA phospholipase $\mathrm{A}_{2}$ (iPLA ${ }_{2} \beta$ ) renders susceptibility for chemical-induced colitis. Dig Dis Sci. (Epub ahead of print). doi:10.1007/s10620-015-3807-5.

8. Maloy KJ, Powrie F. Intestinal homeostasis and its breakdown in inflammatory bowel disease. Nature. 2011;474:298-306.

9. Deuring JJ, de Haar C, Kuipers EJ, Peppelenbosch MP, van der Woude CJ. The cell biology of the intestinal epithelium and its relation to inflammatory bowel disease. Int J Biochem Cell Biol. 2013;45:798-806.

10. Song H, Wohltmann M, Tan M, Ladenson JH, Turk J. Group VIA phospholipase $\mathrm{A}_{2}$ mitigates palmitate-induced $\beta$-cell mitochondrial injury and apoptosis. J Biol Chem. 2014;289:14194-14210.

11. Barbour SE, Nguyen PT, Park M, et al. Group VIA phospholipase $\mathrm{A}_{2}\left(\mathrm{iPLA}_{2} \beta\right)$ modulates $\mathrm{Bcl}-\mathrm{x}$ 5'-splice site selection and suppresses anti-apoptotic Bcl-x(L) in $\beta$-cells. J Biol Chem. 2015; 290:11021-11031.

12. Montrose DC, Nakanishi M, Murphy RC, et al. The role of $\mathrm{PGE}_{2}$ in intestinal inflammation and tumorigenesis. Prostaglandins Other Lipid Mediat. 2015;116-117:26-36. 